(C2010 IEEE. Personal use of this material is permitted. However, permission to reprint/republish this material for advertising or promotional purposes or for creating new collective works for resale or redistribution to servers or lists, or to reuse any copyrighted component of this work in other works must be obtained from the IEEE. 


\section{A Darboux-Frame-Based Formulation of Spin-Rolling Motion of Rigid Objects With Point Contact}

\author{
Lei Cui and Jian S. Dai
}

\begin{abstract}
This paper investigates the kinematics of spin-rolling motion of rigid objects. This paper does not consider slipping but applies a Darboux frame to develop kinematics of spin-rolling motion, which occurs in a nonholonomic system. A new formulation of spin-rolling motion of the moving object is derived in terms of contravariant vectors, rolling velocity, and geometric invariants, including normal curvature, geodesic curvature, and geodesic torsion of the respective contact curve. The equation is represented with geometric invariants. It can be readily generalized to suit both arbitrary parametric surface and contact trajectory and can be differentiated to any order. Effect of the relative curvatures and torsion on spin-rolling kinematics is explicitly presented. The translation velocity of an arbitrary point on the moving object is also derived based on the Darboux frame.
\end{abstract}

Index Terms-Darboux frame, grasping, kinematics, motion, multifingered hand, nonholonomic, point contact, rolling, spin-rolling.

\section{INTRODUCTION}

When a multifingered hand manipulates an object, rolling and slipping often occur at the contact point between the fingertips and the grasped object. Since there are many benefits of the rolling contact, including reduction of abrasion wear, simplification of controller, and enlargement of reachable configurations, rolling contact without slipping is preferred when the grasped object needs fine tuning. Kinematics of rigid objects with rolling contact is a problem of nonholonomic constraint, i.e., the equation that relates two objects is expressed in terms of their velocities rather than positions. Moreover, the equation becomes a function of shapes of the two objects.

Kinematics of the rolling contact can be divided into two broad categories: pure-rolling motion and spin-rolling motion. Pure-rolling motion has 2 degrees of freedom (DOFs). Its instantaneous rotation axis always passes the contact point and is parallel to the common tangent plane of two objects. Spin-rolling motion, which is also called twist-rolling motion, has 3 DOFs. Its instantaneous rotation axis always passes the contact point, but it can be in any arbitrary direction.

Kinematics of a rolling contact was sometimes included as a special case of kinematics of the contact. One exception was the work of Neimark and Fufaev [1], who derived the velocity equation of spinrolling motion based on the frames along the direction of lines of curvature. Kinematics of a point contact between rigid bodies has attracted many researchers. Cai and Roth [2], [3] investigated kinematics of rigid objects in point contact, both in planar and spatial cases, and focused on two special motions, including slipping and pure-rolling motion. Montana [4] studied the kinematics of sliding-spin-rolling from a geometric point of view and derived the equation of contact.

Manuscript received October 13, 2009; revised January 2, 2010. First published February 8, 2010; current version published April 7, 2010. This paper was recommended for publication by Associate Editor W. Chung and Editor J.-P. Laumond upon evaluation of the reviewers' comments.

The authors are with the School of Physics and Engineering, King's College London, University of London, London, WC2R 2LS, U.K. (e-mail: lei.cui@ kcl.ac.uk; jian.s.dai@kcl.ac.uk).

Color versions of one or more of the figures in this paper are available online at http://ieeexplore.ieee.org.

Digital Object Identifier 10.1109/TRO.2010.2040201
Sarkar et al. [5] extended Montana's work to include acceleration terms. By using intrinsic geometric properties for the contact surfaces, they showed the explicit dependence on the Christoffel symbols and their time derivatives. Marigo and Bicchi [6] derived analogous equations with Montana's contact equations but with a different approach that allowed an analysis of admissibility of a pure-rolling contact. Agrachev and Sachkov [7] solved the controllability problem of a pair of purerolling rigid bodies. Chelouah and Chitour [8] addressed the issue of motion planning for the control system that results from pure-rolling of a 2-D Riemannian manifold onto another and presented two procedures to tackle the motion-planning problem when one manifold was a plane and the other a convex surface. Chitour et al. [9] studied the pure-rolling of a pair of smooth convex objects, with one being on top of the other under quantized control. Tchon [10] introduced and examined the property of repeatability of inverse-kinematics algorithms for mobile manipulators. Tchon and Jakubiak [11] derived an extendedJacobian-inverse-kinematics algorithm for doubly nonholonomic mobile manipulators based on the concept of endogenous configuration space. Li and Canny [12] used Montana's contact equations to study whether an admissible path exists between two configurations in the case of pure rolling and, if it does, then how to find it.

It is acknowledged that rolling and the curvature effect on it are essential in the design of simple and dexterous hands. Hence, it is not surprising to see that much of the literature that has appeared in recent years is related to grasping and manipulation of objects and control of multifingered hands that concern kinematics of rolling contact between fingertips and the object. Ghafoor et al. [13], [14] simplified kinematics of rolling contact to line contact to study stiffness modeling and finemotion control of the interaction between fingertips and the grasped object. Kerr and Roth [15] discussed how to compute the movement of the fingers in order to produce a given displacement of the object. Remond et al. [16] acknowledged the need of rolling compensation for multifingered-hand control structure and proposed polar parameterization of the local shape around the contact point to get four independent equations. Some other literature regarding motion planning and control of the multifingered robotic hand can be found in [17]-[26].

However, the aforementioned mathematical derivation of kinematics of rolling contact suffers from some drawbacks. First, the derivation of equations is generally difficult to obtain; as stated in [16], it cannot be easily done in general. Second, it is local in nature. In other words, it depends on the parameterization of the contact surfaces. If either the origin or the orientation of the global coordinate changes, the formulations are no longer valid. Third, the formulations can be differentiated only to a certain order, which is usually either one or two.

The main contribution of this paper is that a new equation of the angular velocity of the spin-rolling motion of the moving object is derived. The new formulation is expressed in terms of three contravariant vectors and geometric invariants, which are arc length of the contacttrajectory curve and the induced curvatures of the two surfaces.

A contravariant vector is a geometric entity whose components will be transformed in a certain way that passes from one coordinate system to another. The contravariant vector itself does not change. However, the components of the contravariant vector might vary due to the change of a coordinate system. In the language of tensor analysis, a contravariant vector is a type $(1,0)$ tensor.

The invariants include the magnitude of rolling velocity and the induced curvatures of the two surfaces. These invariants remain identical, regardless of coordinate transformations. 


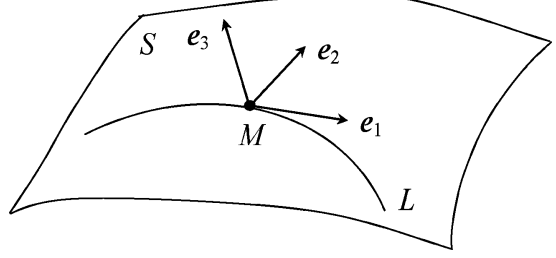

Fig. 1. Darboux frame at point $M$.

If the new contravariant-vector-based angular velocity is formulated in a coordinate system, change of the coordinate system results only in the change of components of contravariant vectors, and it does not change the formulation. In this sense, the formulation proposed in this paper is coordinate-invariant.

Thus, the equation can be formulated in a convenient local coordinate system and can later be used in a global one. Further, the equation is represented in terms of geometric invariants. It can be readily generalized to suit both arbitrary parametric surface and contact trajectory and can be differentiated to any order. The effects of the relative curvature and torsion on spin-rolling motion are explicitly presented in this equation.

This paper is organized as follows. Section II introduces the concept of Darboux frame. Section III investigates geometric kinematics of spin-rolling motion. Section IV obtains a new equation of the angular velocity of the spin-rolling motion in terms of the rolling speed and two sets of geometric invariants. Section $\mathrm{V}$ gives two examples that show how to apply the proposed method. Finally, Section VI concludes this paper.

\section{DARBOUX FRAME}

A brief introduction that concerns some basic concepts of differential geometry is given in this section. Details can be found in [27]-[29].

Let $L$ be an oriented curve that is traced on an oriented surface $S$ in a 3-D Euclidean space, and Darboux frame $\left\{\boldsymbol{e}_{1}, \boldsymbol{e}_{2}, \boldsymbol{e}_{3}\right\}$ is a right-handed orthogonal frame that is associated with each point of $M \in L$, as shown in Fig. 1 , where $\boldsymbol{e}_{1}$ is the unit tangent vector to $L, \boldsymbol{e}_{3}$ is the unit normal to $S, \boldsymbol{e}_{2}$ is tangential to $S$, and $\boldsymbol{e}_{2}=\boldsymbol{e}_{3} \times \boldsymbol{e}_{1}$ at point $M$, where $\boldsymbol{e}_{1}, \boldsymbol{e}_{2}$, and $\boldsymbol{e}_{3}$ are row vectors.

The equations of motion of the Darboux frame can be written as

$$
\left\{\begin{array}{l}
\frac{d \boldsymbol{M}}{d s}=\boldsymbol{e}_{1} \\
\frac{d}{d s}\left[\begin{array}{l}
\boldsymbol{e}_{1} \\
\boldsymbol{e}_{2} \\
\boldsymbol{e}_{3}
\end{array}\right]=\left[\begin{array}{ccc}
0 & k_{g} & k_{n} \\
-k_{g} & 0 & \tau_{g} \\
-k_{n} & -\tau_{g} & 0
\end{array}\right]\left[\begin{array}{l}
\boldsymbol{e}_{1} \\
\boldsymbol{e}_{2} \\
\boldsymbol{e}_{3}
\end{array}\right]
\end{array}\right.
$$

where $s$ is the arc length of curve $L$, and $k_{q}, k_{n}$, and $\tau_{g}$ are the geodesic curvature, the normal curvature, and the geodesic torsion, respectively.

The vector $M$ in (1) is a position vector that depends on the choice of the coordinate system. The components of the vector $M$ are obtained from the measurement along the axes of the coordinate system.

However, the three vectors $\boldsymbol{e}_{1}, \boldsymbol{e}_{2}$, and $\boldsymbol{e}_{3}$ in (1) are either contravariant vectors or type $(1,0)$ tensors. They do not have any intrinsic coordinate system. The components of these contravariant vectors will transform in a certain way that passes from one coordinate system to another.

The four scalars, i.e., $s, k_{g}, k_{n}$, and $\tau_{g}$ in (1), are geometric invariants that maintain the identical values, regardless of coordinate transformations.

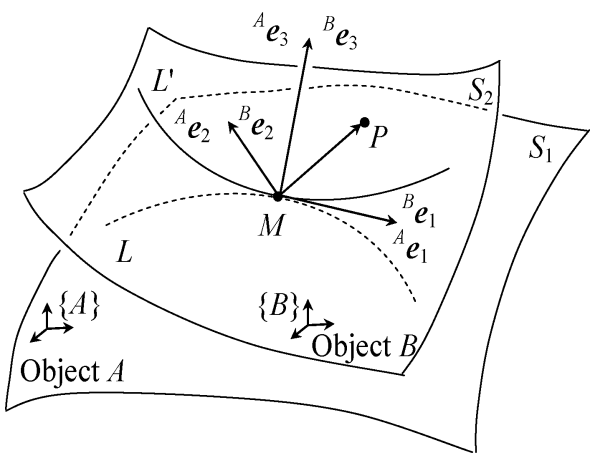

Fig. 2. Object $B$ spin-rolling on object $A$ along curves $L^{\prime}$ and $L$.

\section{Geometric Kinematics of Spin-Rolling Motion}

Assume that objects $A$ and $B$ undergo spin-rolling motion without slipping at every moment. Curve $L$ is the contact-trajectory curve on surface $S_{1}$ of object $A$ and curve $L^{\prime}$ on surface $S_{2}$ of object $B$. Since the aim of this paper is to study the relative motion between the two objects, object $A$ can be assumed to be fixed. Object $B$ has spin-rolling motion relative to object $A$. With the setup frame $\{A\}$ fixed on object $A$ and frame $\{B\}$ fixed on object $B$, a superscript $A$ or $B$ will be used to represent the frame in which a vector is expressed. The moving frame attached to the contact point $M$ of curve $L$ is $\left\{{ }^{A} \boldsymbol{e}_{1},{ }^{A} \boldsymbol{e}_{2},{ }^{A} \boldsymbol{e}_{3}\right\}$, and the frame attached to the contact point $M$ of $L^{\prime}$ is $\left\{{ }^{B} \boldsymbol{e}_{1},{ }^{B} \boldsymbol{e}_{2},{ }^{B} \boldsymbol{e}_{3}\right\}$. Contravariant vectors ${ }^{A} \boldsymbol{e}_{3}$ and ${ }^{B} \boldsymbol{e}_{3}$ are the unit normals of surfaces $S_{1}$ and $S_{2}$, respectively. Contravariant vectors ${ }^{A} \boldsymbol{e}_{1}$ and ${ }^{B} \boldsymbol{e}_{1}$ are the unit tangents to curves $L$ and $L^{\prime}$, respectively. Both $\left\{{ }^{A} \boldsymbol{e}_{1},{ }^{A} \boldsymbol{e}_{2},{ }^{A} \boldsymbol{e}_{3}\right\}$ and $\left\{{ }^{B} \boldsymbol{e}_{1},{ }^{B} \boldsymbol{e}_{2},{ }^{B} \boldsymbol{e}_{3}\right\}$ are right-handed orthonormal frames. Due to rolling constraints, contravariant vectors ${ }^{A} \boldsymbol{e}_{1}$ and ${ }^{B} \boldsymbol{e}_{1}$ are always collinear and, therefore, are ${ }^{A} \boldsymbol{e}_{3}$ and ${ }^{B} \boldsymbol{e}_{3}$. Hence, the two frames can always be made to coincide, as shown in Fig. 2, where contravariant vector ${ }^{A} \boldsymbol{e}_{3}$ points outward of surface $S_{1}$, and ${ }^{B} \boldsymbol{e}_{3}$ points inward of surface $S_{2}$.

Assume that both curves $L$ and $L^{\prime}$ are parameterized by arc length. Let $s$ and $s^{\prime}$ denote the arc lengths of $L$ and $L^{\prime}$, respectively. Let $P$ denote an arbitrary point of object $B$; then, the position vector of point $P$ can be expressed in frame $\{B\}$ as

$$
{ }^{B} \boldsymbol{P}={ }^{B} \boldsymbol{M}+u_{1}^{\prime B} \boldsymbol{e}_{1}+u_{2}^{\prime B} \boldsymbol{e}_{2}+u_{3}^{\prime B} \boldsymbol{e}_{3} .
$$

Differentiating (2) with respect to $s^{\prime}$ yields

$$
\begin{aligned}
\frac{d^{B} \boldsymbol{P}}{d s^{\prime}}= & \left(1+\frac{d u_{1}^{\prime}}{d s^{\prime}}-u_{2}^{\prime} k_{g}^{\prime}-u_{3}^{\prime} k_{n}^{\prime}\right){ }^{B} \boldsymbol{e}_{1}+\left(\frac{d u_{2}^{\prime}}{d s^{\prime}}+u_{1}^{\prime} k_{g}^{\prime}-u_{3}^{\prime} \tau_{g}^{\prime}\right)^{B} \boldsymbol{e}_{2} \\
& +\left(\frac{d u_{3}^{\prime}}{d s^{\prime}}+u_{1}^{\prime} k_{n}^{\prime}+u_{2}^{\prime} \tau_{g}^{\prime}\right)^{B} \boldsymbol{e}_{3}
\end{aligned}
$$

where $k_{g}^{\prime}, k_{n}^{\prime}$, and $\tau_{g}^{\prime}$ are the geodesic curvature, the normal curvature, and the geodesic torsion at point $M$ of $L^{\prime}$. Since $P$ is a fixed point of object $B$, it follows that

$$
\frac{d^{B} \boldsymbol{P}}{d s^{\prime}}=0
$$

Substituting this into (3) yields

$\frac{d u_{1}^{\prime}}{d s^{\prime}}=u_{2}^{\prime} k_{g}^{\prime}+u_{3}^{\prime} k_{n}^{\prime}-1, \frac{d u_{2}^{\prime}}{d s^{\prime}}=-u_{1}^{\prime} k_{g}^{\prime}+u_{3}^{\prime} \tau_{g}^{\prime}, \frac{d u_{3}^{\prime}}{d s^{\prime}}=-u_{1}^{\prime} k_{n}^{\prime}-u_{2}^{\prime} \tau_{g}^{\prime}$.

Point $P$ can also be expressed in frame $\{A\}$ as

$$
{ }^{A} \boldsymbol{P}={ }^{A} \boldsymbol{M}+u_{1}{ }^{A} \boldsymbol{e}_{1}+u_{2}{ }^{A} \boldsymbol{e}_{2}+u_{3}{ }^{A} \boldsymbol{e}_{3} .
$$


Differentiating (6) with respect to $s$ yields

$$
\begin{aligned}
\frac{d^{A} \boldsymbol{P}}{d s}= & \left(1+\frac{d u_{1}}{d s}-u_{2} k_{g}-u_{3} k_{n}\right){ }^{A} \boldsymbol{e}_{1}+\left(\frac{d u_{2}}{d s}+u_{1} k_{g}-u_{3} \tau_{g}\right){ }^{A} \boldsymbol{e}_{2} \\
& +\left(\frac{d u_{3}}{d s}+u_{1} k_{n}+u_{2} \tau_{g}\right){ }^{A} \boldsymbol{e}_{3}
\end{aligned}
$$

where $k_{g}, k_{n}$, and $\tau_{g}$ are the geodesic curvature, the normal curvature, and the geodesic torsion at point $M$ of curve $L$. The rolling constraints require that the velocities of the two contact curves be equal, which results in the arc lengths of the two contact-trajectory curves covered in the same time period being identical, i.e., $s=s^{\prime}$. Since moving frames $\left\{{ }^{A} \boldsymbol{e}_{1},{ }^{A} \boldsymbol{e}_{2},{ }^{A} \boldsymbol{e}_{3}\right\}$ and $\left\{{ }^{B} \boldsymbol{e}_{1},{ }^{B} \boldsymbol{e}_{2},{ }^{B} \boldsymbol{e}_{3}\right\}$ are made to coincide at any moment, it follows that

$$
u_{1}=u_{1}^{\prime}, u_{2}=u_{2}^{\prime}, u_{3}=u_{3}^{\prime}
$$

and consequently

$$
\frac{d u_{1}}{d s}=\frac{d u_{1}^{\prime}}{d s^{\prime}}, \frac{d u_{2}}{d s}=\frac{d u_{2}^{\prime}}{d s^{\prime}}, \frac{d u_{3}}{d s}=\frac{d u_{3}^{\prime}}{d s^{\prime}} .
$$

Thus, due to the coincidence, $s$ can be used to denote both $s$ and $s^{\prime}$, and $u_{i}$ can be used to denote both $u_{i}$ and $u_{i}{ }^{\prime}$. Substituting (3) and (9) into (7) yields

$$
\begin{aligned}
\frac{d^{A} \boldsymbol{P}}{d s}= & \left(u_{2} k_{g}^{*}+u_{3} k_{n}^{*}\right)^{A} \boldsymbol{e}_{1}+\left(-u_{1} k_{g}^{*}+u_{3} \tau_{g}^{*}\right)^{A} \boldsymbol{e}_{2} \\
& +\left(-u_{1} k_{n}^{*}-u_{2} \tau_{g}^{*}\right)^{A} \boldsymbol{e}_{3}
\end{aligned}
$$

where

$$
k_{g}^{*}=k_{g}^{\prime}-k_{g}, \quad k_{n}^{*}=k_{n}^{\prime}-k_{n}, \quad \tau_{g}^{*}=\tau_{g}^{\prime}-\tau_{g}
$$

are called induced geodesic curvature, normal curvature, and geodesic torsion, respectively.

\section{DARBOUX-Frame-BASED VElOCITY Formulation OF SPIN-ROLLING MOTION}

If time $t$ is considered, the velocity of an arbitrary point $P$ on object $B$ can be obtained from (10) as follows:

$$
\begin{aligned}
{ }^{A} \boldsymbol{v}_{P}= & \frac{d^{A} \boldsymbol{P}}{d s} \frac{d s}{d t}=\sigma\left(u_{2} k_{g}^{*}+u_{3} k_{n}^{*}\right){ }^{A} \boldsymbol{e}_{1} \\
& +\sigma\left(u_{3} \tau_{g}^{*}-u_{1} k_{g}^{*}\right){ }^{A} \boldsymbol{e}_{2}-\sigma\left(u_{1} k_{n}^{*}+u_{2} \tau_{g}^{*}\right){ }^{A} \boldsymbol{e}_{3}
\end{aligned}
$$

where $\sigma=d s / d t$ is the magnitude of rolling velocity. This gives the Darboux-frame-based translational-velocity formulation of an arbitrary point. On the other hand, suppose the angular velocity of object $B$ with respect to object $A$ is

$$
{ }^{A} \boldsymbol{\omega}=\omega_{x}{ }^{A} \boldsymbol{e}_{1}+\omega_{y}{ }^{A} \boldsymbol{e}_{2}+\omega_{z}{ }^{A} \boldsymbol{e}_{3} .
$$

The velocity of point $P$ can also be obtained as

$$
\begin{aligned}
{ }^{A} \boldsymbol{v}_{P}= & { }^{A} \boldsymbol{\omega} \times{ }^{A} \boldsymbol{r}_{M P}=\left(-u_{2} \omega_{z}+u_{3} \omega_{y}\right){ }^{A} \boldsymbol{e}_{1} \\
& +\left(u_{1} \omega_{z}-u_{3} \omega_{x}\right)^{A} \boldsymbol{e}_{2}+\left(-u_{1} \omega_{y}+u_{2} \omega_{x}\right)^{A} \boldsymbol{e}_{3}
\end{aligned}
$$

where ${ }^{A} \boldsymbol{r}_{M P}=u_{1}{ }^{A} \boldsymbol{e}_{1}+u_{2}{ }^{A} \boldsymbol{e}_{2}+u_{3}{ }^{A} \boldsymbol{e}_{3}$. Comparing (12) with (14) gives

$$
\omega_{x}=-\sigma \tau_{g}^{*}, \quad \omega_{y}=\sigma k_{n}^{*}, \quad \omega_{z}=-\sigma k_{g}^{*} .
$$

The angular velocity of object $B$ is, thus, obtained from (13) and (15) as

$$
{ }^{A} \boldsymbol{\omega}=\sigma\left(-\tau_{g}^{* A} \boldsymbol{e}_{1}+k_{n}^{* A} \boldsymbol{e}_{2}-k_{g}^{* A} \boldsymbol{e}_{3}\right) .
$$

This gives the Darboux-frame-based angular velocity of spin-rolling motion of the moving object. There are three terms in (16), where the first two terms, i.e., $-\sigma \tau_{g}^{* A} \boldsymbol{e}_{1}+\sigma k_{n}^{* A} \boldsymbol{e}_{2}$, give the pure-rolling velocity about an axis in the tangent plane at the contact point, and the third term, i.e., $-\sigma k_{g}^{* A} \boldsymbol{e}_{3}$, gives the velocity of spin motion about the normal direction at the contact point.

It can be seen that the magnitude of rolling velocity $\sigma$ and the induced curvatures $\tau_{g}^{*}, k_{n}^{*}$, and $k_{g}^{*}$ are scalars, and ${ }^{A} \boldsymbol{e}_{i}$ are contravariant vectors. Since a contravariant vector has a direction and a magnitude, and it has no intrinsic coordinates, the equation of the angular velocity can be formulated in a convenient local coordinate frame and can later be used in a global frame without change. In this sense, (16) is coordinateinvariant.

Another advantage is that (16) is represented in geometric invariants that can be readily generalized to arbitrary parametric surfaces and contact trajectories. Higher order kinematics of the moving object can be obtained by differentiating the right-hand side of (16).

Together with the translational velocity of (12), the kinematics of the spin-rolling motion of the moving object is determined by these two Darboux-frame-based equations.

A pure-rolling motion does not have spin motion in the direction of the normal of the surfaces. It requires that

$$
k_{g}=k_{g}^{\prime} .
$$

Thus, the following corollary can be concluded.

Corollary 1: If two objects undergo pure-rolling motion, the values of the geodesic curvature of the two corresponding contact-trajectory curves have to be identical.

This conclusion corresponds to that reported in [9], in which neither rolling with neither slipping nor spinning was defined in terms of Darboux frame.

To control the moving object to follow the desired trajectory curve on the fixed object, the first two terms of (16) provide the information about the angular velocity in the direction parallel to the tangent plane. The third term of (16) provides the angular velocity of the spin motion. This information can be used as input of the control system to make the moving object follow the desired trajectory curve on the fixed object.

\section{EXAMPLES}

In this section, two examples are provided to show how the proposed method can be applied. The first example shows the reconciliation of the velocity formulation in this paper with a classical equation and also the advantages of the proposed formulation. The second example demonstrates how the equation can be obtained when the arc lengths of the contact-trajectory curves do not have a closed-form solution, and their geometric invariants are not constant.

\section{A. Reconciliation of the Velocity Formulation With the Classical Equation}

Consider the classical example of a disk of radius $R$ spin-rolling on the plane, as shown in Fig. 3, and the disk remains upright. The contact curves are the circle $L^{\prime}$ of the disk and the curve $L$ in the plane. In this case, the disk can be considered to be either a degenerated surface or a ball, with a big circle as the contact curve.

Suppose the curvature of $L$ is $k$ and that ${ }^{A} \boldsymbol{e}_{3}$ is in the outward direction of the plane. The Darboux equations of $L$ are

$$
\frac{d}{d s}\left[\begin{array}{l}
{ }^{A} \boldsymbol{e}_{1} \\
{ }^{A} \boldsymbol{e}_{2} \\
{ }^{A} \boldsymbol{e}_{3}
\end{array}\right]=\left[\begin{array}{ccc}
0 & k & 0 \\
-k & 0 & 0 \\
0 & 0 & 0
\end{array}\right]\left[\begin{array}{l}
{ }^{A} \boldsymbol{e}_{1} \\
{ }^{A} \boldsymbol{e}_{2} \\
{ }^{A} \boldsymbol{e}_{3}
\end{array}\right]
$$




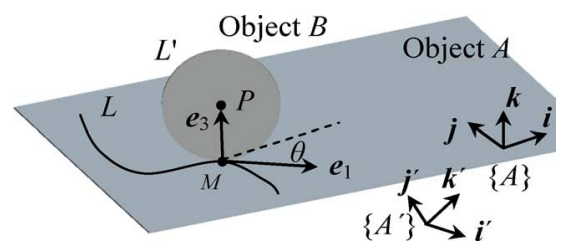

Fig. 3. Disk spin-rolling on a plane.

Let the unit normal ${ }^{B} \boldsymbol{e}_{3}$ of the disk be inward; then, the Darboux equations of $L^{\prime}$ are

$$
\frac{d}{d s}\left[\begin{array}{l}
{ }^{B} \boldsymbol{e}_{1} \\
{ }^{B} \boldsymbol{e}_{2} \\
{ }^{B} \boldsymbol{e}_{3}
\end{array}\right]=\left[\begin{array}{ccc}
0 & 0 & 1 / R \\
0 & 0 & 0 \\
-1 / R & 0 & 0
\end{array}\right]\left[\begin{array}{l}
{ }^{B} \boldsymbol{e}_{1} \\
{ }^{B} \boldsymbol{e}_{2} \\
{ }^{B} \boldsymbol{e}_{3}
\end{array}\right] .
$$

From Darboux-frame-based velocity formulation (16), it follows that the angular velocity of the disk is

$$
{ }^{A} \boldsymbol{\omega}=\sigma\left(\frac{1}{R}{ }^{A} \boldsymbol{e}_{2}-k^{A} \boldsymbol{e}_{3}\right)
$$

The coordinate of the center point $P$ of the disk in moving frame $\left\{{ }^{A} \boldsymbol{e}_{1},{ }^{A} \boldsymbol{e}_{2},{ }^{A} \boldsymbol{e}_{3}\right\}$ at point $M$ is $(0,0, R)$. From Darboux-frame-based translation formulation (12) and its alternation (14), it follows that the velocity of point $P$ is

$$
{ }^{A} \boldsymbol{v}_{P}={ }^{A} \boldsymbol{\omega} \times{ }^{A} \boldsymbol{r}_{M P}=\sigma\left(\frac{1}{R}{ }^{A} \boldsymbol{e}_{2}-k^{A} \boldsymbol{e}_{3}\right) \times R^{A} \boldsymbol{e}_{3}=\sigma{ }^{A} \boldsymbol{e}_{1} .
$$

In textbooks about dynamics, such as those by Rosenberg [30] and Bullo and Lewis [31], four general coordinates $(x, y, \theta, \varphi)$ were taken to study this contact. For this purpose, it is necessary to establish a coordinate system $\{A-\boldsymbol{i} \boldsymbol{j} \boldsymbol{k}\}$ with point $A$ on the plane, $\boldsymbol{i}$ in the plane, $\boldsymbol{k}$ perpendicular to the plane, and $\boldsymbol{i}-\boldsymbol{j}-\boldsymbol{k}$ being an orthogonal frame, as shown in Fig. 3. The angular velocity of the disk and the velocity of the centerpoint of the disk are, respectively, given by

$$
\begin{aligned}
{ }^{A} \boldsymbol{\omega} & =\frac{d \varphi}{d t} \cos \theta \boldsymbol{i}+\frac{d \varphi}{d t} \sin \theta \boldsymbol{j}-\frac{d \theta}{d t} \boldsymbol{k} \\
{ }^{A} \boldsymbol{v}_{P} & =R \frac{d \varphi}{d t} \cos \theta \boldsymbol{i}+R \frac{d \varphi}{d t} \sin \theta \boldsymbol{j} .
\end{aligned}
$$

If the replacements

$$
\begin{aligned}
{ }^{A} \boldsymbol{e}_{1} & =\cos \theta \boldsymbol{i}+\sin \theta \boldsymbol{j}, \quad{ }^{A} \boldsymbol{e}_{2}=-\sin \theta \boldsymbol{i}+\cos \theta \boldsymbol{j}, \quad{ }^{A} \boldsymbol{e}_{3}=\boldsymbol{k} \\
\sigma & =R \frac{d \varphi}{d t}=\frac{d s}{d t}, \quad k=\frac{d \theta}{d s} .
\end{aligned}
$$

are substituted into (20) and (21), it can be seen that (20) and (21) are the same as (22), and the Darboux-frame-based velocity formulation reconciles with the classical velocity formulation.

The advantages of the proposed formulation can be seen in the following discussion. If the angular velocity is formulated in the ways that have been proposed in previous literature, and if both the origin and orientation of the coordinate system change from $\{A-\boldsymbol{i j} \boldsymbol{k}\}$ to $\left\{A^{\prime}-\boldsymbol{i}^{\prime} \boldsymbol{j}^{\prime} \boldsymbol{k}^{\prime}\right\}$, as shown in Fig. 3, the angular formulation in (22) must be reestablished.

In contrast, if both the origin and the orientation of the coordinate system change from $\{A-\boldsymbol{i} \boldsymbol{j} \boldsymbol{k}\}$ to $\left\{A^{\prime}-\boldsymbol{i}^{\prime} \boldsymbol{j}^{\prime} \boldsymbol{k}^{\prime}\right\}$, the angular velocity (20) derived from the proposed approach becomes

$$
{ }^{A^{\prime}} \boldsymbol{\omega}=\sigma\left(\frac{1}{R}{ }^{A^{\prime}} \boldsymbol{e}_{2}-k^{A^{\prime}} \boldsymbol{e}_{3}\right)
$$

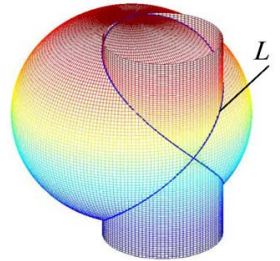

(a)

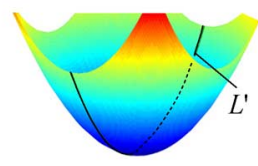

(b)
Fig. 4. (a) Intersection of a cylinder and a sphere. (b) One meridian of the surface of revolution.

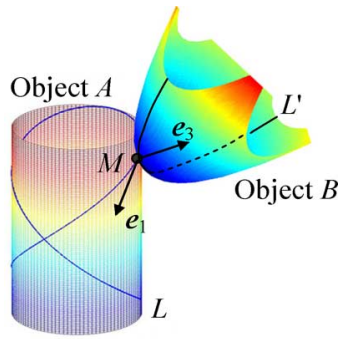

Fig. 5. Object $B$ spin-rolling on object $A$ along curves $L^{\prime}$ and $L$.

It can be seen that only the components of the contravariant vectors ${ }^{A} \boldsymbol{e}_{2}$ change to ${ }^{A^{\prime}} \boldsymbol{e}_{2}$ and ${ }^{A} \boldsymbol{e}_{3}$ to ${ }^{A^{\prime}} \boldsymbol{e}_{3}$. The formulation remains the same. In this sense, the proposed formulation is coordinate-invariant.

Next, a brief discussion is provided using the information from the velocity formulation to control object $B$ to follow the desired trajectory curve $L$ on the fixed object $A$. Object $B$ has 2 DOFs. At any instant, the first term $\sigma / R$ of (20) provides the angular velocity about the axis that is perpendicular to the disk, and the second term $-\sigma k$ provides the information about how fast the disk spins to follow the curve $L$ and, thus, gives the new tangent direction of the trajectory $L$. This information can be used as the inputs of the control system to make object $B$ follow the trajectory curve $L$.

\section{B. Application to the Spin-Rolling Motion With Variable Geometric Invariants}

It is rare for a curve to have a closed-form solution of its arc length. It can be concluded that geometric invariants are obtainable if the arc length can be differentiated.

The surface of object $A$ in this section is a cylinder of radius $R$, and curve $L$ is the intersecting curve of the cylinder and a sphere of radius $2 R$, as shown in Fig. 4(a). The surface of object $B$ is a surface of revolution that is formed by rotating a parabola $z=1 / 2 y^{2}$ around $z$-axis, and curve $L^{\prime}$ is that of the meridians, as shown in Fig. 4(b).

Now, suppose object $B$ rolls without slipping on object $A$ along curves $L$ and $L^{\prime}$ of objects $A$ and $B$, respectively, where point $M$ is the contact point, as shown in Fig. 5.

Curve $L$ can be parameterized as

$$
{ }^{A} \boldsymbol{L}(\theta)=R\left(\cos \theta, \sin \theta, 2 \sin \frac{\theta}{2}\right) .
$$

The differential of arc length $s$ of curve $L$ with respect to $\theta$ can be obtained as

$$
\frac{d s}{d \theta}=\sqrt{\frac{d^{A} \boldsymbol{L}}{d \theta} \frac{d^{A} \boldsymbol{L}}{d \theta}}=R \sqrt{1+\cos ^{2} \theta} .
$$


The arc length $s$ does not admit a closed-form solution. It follows that the unit tangent vector ${ }^{A} \boldsymbol{e}_{1}$ can be given by

$$
{ }^{A} \boldsymbol{e}_{1}=\frac{d^{A} \boldsymbol{L}}{d s}=\frac{d^{A} \boldsymbol{L}}{d \theta} / \frac{d s}{d \theta}=\frac{(-\sin \theta, \cos \theta, \cos (\theta / 2))}{\sqrt{1+\cos ^{2}(\theta / 2)}} .
$$

Let the unit normal ${ }^{A} \boldsymbol{e}_{3}$ of object $A$ be outward, which can be obtained as

$$
{ }^{A} \boldsymbol{e}_{3}=(\cos \theta, \sin \theta, 0) .
$$

After the algebraic operation, the geodesic curvature, the normal curvature, and the geodesic torsion of curve $L$ can be obtained as

$$
\begin{aligned}
k_{g} & =\left(\frac{d^{A} \boldsymbol{e}_{1}}{d \theta} / \frac{d s}{d \theta}\right){ }^{A} \boldsymbol{e}_{2}=\frac{-\sin (\theta / 2)}{2 R\left(1+\cos ^{2}(\theta / 2)\right)^{3 / 2}} \\
k_{n} & =\left(\frac{d^{A} \boldsymbol{e}_{1}}{d \theta} / \frac{d s}{d \theta}\right){ }^{A} \boldsymbol{e}_{3}=\frac{-1}{R\left(1+\cos ^{2}(\theta / 2)\right)} \\
\tau_{g} & =\left(\frac{d^{A} \boldsymbol{e}_{2}}{d \theta} / \frac{d s}{d \theta}\right){ }^{A} \boldsymbol{e}_{3}=\frac{\cos (\theta / 2)}{R\left(1+\cos ^{2}(\theta / 2)\right)} .
\end{aligned}
$$

However, curve $L^{\prime}$ can be parameterized as

$$
{ }^{B} \boldsymbol{L}^{\prime}(s)=\left(u, v_{0}, \frac{1}{2}\left(u^{2}+v_{0}^{2}\right)\right)
$$

where $v_{0}$ is a constant. This makes curve $L^{\prime}$ a meridian of surface of object $B$. The differential of arc length $s^{\prime}$ of curve $L^{\prime}$ with respect to $u$ can be obtained as

$$
\frac{d s^{\prime}}{d u}=\sqrt{\frac{d^{B} \boldsymbol{L}^{\prime}}{d u} \frac{d^{B} \boldsymbol{L}^{\prime}}{d u}}=\sqrt{u^{2}+1} .
$$

The unit tangent vector ${ }^{B} \boldsymbol{e}_{1}$ of curve $L^{\prime}$ can be obtained as

$$
{ }^{B} \boldsymbol{e}_{1}=\frac{d^{B} \boldsymbol{L}^{\prime}}{d s^{\prime}}=\frac{d^{B} \boldsymbol{L}^{\prime}}{d u} / \frac{d s^{\prime}}{d u}=\frac{1}{\sqrt{u^{2}+1}}(1,0, u) .
$$

Let the unit normal ${ }^{B} \boldsymbol{e}_{3}$ of object $B$ be inward, and it can be obtained as

$$
{ }^{B} \boldsymbol{e}_{3}=\frac{1}{\sqrt{u^{2}+v_{0}^{2}+1}}\left(u, v_{0},-1\right) .
$$

After some algebraic manipulation, the geodesic curvature, the normal curvature, and the geodesic torsion of curve $L^{\prime}$ can be obtained as

$$
\begin{aligned}
k_{g}^{\prime} & =\left(\frac{d^{B} \boldsymbol{e}_{1}}{d u} / \frac{d s^{\prime}}{d u}\right){ }^{B} \boldsymbol{e}_{2}=\frac{-v_{0}}{\left(u^{2}+1\right)^{3 / 2} \sqrt{u^{2}+v_{0}^{2}+1}} \\
k_{n}^{\prime} & =\left(\frac{d^{B} \boldsymbol{e}_{1}}{d u} / \frac{d s^{\prime}}{d u}\right)^{B} \boldsymbol{e}_{3}=\frac{-1}{\left(u^{2}+1\right) \sqrt{u^{2}+v_{0}^{2}+1}} \\
\tau_{g}^{\prime} & =\left(\frac{d^{B} \boldsymbol{e}_{2}}{d u} / \frac{d s^{\prime}}{d u}\right)^{B} \boldsymbol{e}_{3}=\frac{-u v_{0}}{\left(u^{2}+1\right)\left(u^{2}+v_{0}^{2}+1\right)} .
\end{aligned}
$$

From (16), the angular velocity of object $B$ can be obtained as

$$
\begin{aligned}
{ }^{A} \boldsymbol{\omega}= & \sigma\left(\frac{u v_{0}}{\left(u^{2}+1\right)\left(u^{2}+v_{0}^{2}+1\right)}+\frac{\cos (\theta / 2)}{R\left(1+\cos ^{2}(\theta / 2)\right)}\right){ }^{A} \boldsymbol{e}_{1} \\
& +\sigma\left(\frac{-1}{\left(u^{2}+1\right) \sqrt{u^{2}+v_{0}^{2}+1}}+\frac{1}{R\left(1+\cos ^{2}(\theta / 2)\right)}\right){ }^{A} \boldsymbol{e}_{2} \\
& +\sigma\left(\frac{v_{0}}{\left(u^{2}+1\right)^{3 / 2} \sqrt{u^{2}+v_{0}^{2}+1}}-\frac{\sin (\theta / 2)}{2 R\left(1+\cos ^{2}(\theta / 2)\right)^{3 / 2}}\right){ }^{A} \boldsymbol{e}_{3} .
\end{aligned}
$$

From the derivation in this example, it can be seen that the proposed method is expressed in terms of geometric invariants that can be readily applied to arbitrary parametric surfaces and curves.

\section{CONCLUSION}

This paper adopted the moving-frame method to study the instantaneous spin-rolling motion of rigid objects. The rolling constraints require that velocities of the two contact curves be equal, which results in the invariant arc lengths of the two contact-trajectory curves being identical in the same period of time. This geometric constraint facilitated subsequent analysis. It was found that the magnitude of rolling velocity and two sets of geometric invariants, including the geodesic curvature, the normal curvature, and the geodesic torsion, determine the instantaneous kinematics of a moving object. The result was expressed in terms of invariants that could be readily generalized to arbitrary parametric surfaces and contact curves. The effects of the relative curvature and torsions on spin-rolling kinematics were presented explicitly. It is hoped that new light can be shed on dexterous manipulations and motion planning.

\section{REFERENCES}

[1] I. I. Neimark and N. A. Fufaev, Dynamics of Nonholonomic Systems. Providence, RI: Amer. Math. Soc., 1972.

[2] C. Cai and B. Roth, "On the planar motion of rigid bodies with point contact," Mech. Mach. Theory, vol. 21, no. 6, pp. 453-466, 1986.

[3] C. Cai and B. Roth, "On the spatial motion of rigid bodies with point contact," in Proc. IEEE Conf. Robot. Autom., 1987, pp. 686-695.

[4] D. J. Montana, "The kinematics of contact and grasp," Int. J. Robot. Res., vol. 7, no. 3, pp. 17-32, 1988.

[5] N. Sarkar, V. Kumar, and X. Yun, "Velocity and acceleration equations for three-dimensional contact," J. Appl. Mech., vol. 63, no. 4, pp. 974-984 1996.

[6] A. Marigo and A. Bicchi, "Rolling bodies with regular surface: Controllability theory and application," IEEE Trans. Autom. Control, vol. 45, no. 9, pp. 1586-1599, Sep. 2000.

[7] A. A. Agrachev and Y. L. Sachkov, "An intrinsic approach to the control of rolling bodies," in Proc. 38th IEEE Conf. Decis. Control, Phoenix, AZ, 1999, pp. 431-435.

[8] A. Chelouah and Y. Chitour, "On the motion planning of rolling surfaces," Forum Math., vol. 15, no. 5, pp. 727-758, 2003.

[9] Y. Chitour, A. Marigo, and B. Piccoli, "Quantization of the rolling-body problem with applications to motion planning," Syst. Control Lett., vol. 54, no. 10, pp. 999-1013, 2005.

[10] K. Tchon, "Repeatability of inverse kinematics algorithms for mobile manipulators," IEEE Trans. Autom. Control, vol. 47, no. 8, pp. 13761380, Aug. 2002.

[11] K. Tchon and J. Jakubiak, "An extended Jacobian inverse kinematics algorithm for doubly nonholonomic mobile manipulators," in Proc. IEEE Int. Conf. Robot. Autom., Barcelona, Spain, 2005, pp. 1548-1553.

[12] Z.X. Li and J. Canny, "Motion of two rigid bodies with rolling constraint," IEEE Trans. Robot. Autom., vol. 6, no. 1, pp. 62-72, Feb. 1990

[13] A. Ghafoor, J. S. Dai, and J. Duffy, "Stiffness modeling of a soft-finger contact in robotic grasping," Trans. ASME, J. Mech. Des., vol. 126, no. 4, pp. 646-656, 2004.

[14] A. Ghafoor, J. S. Dai, and J. Duffy, "Fine motion control based on constraint criteria under pre-loading configurations," J. Robot. Syst., vol. 17, no. 4, pp. 171-185, 2000.

[15] J. Kerr and B. Roth, "Analysis of multifingered hands," Int. J. Robot. Res., vol. 4, no. 4, pp. 3-17, 1986.

[16] C. Remond, V. Perdereau, and M. Drourin, "A hierarchical multi-fingered hand control structure with rolling contact compensation," in Proc. IEEE Int. Conf. Robot. Autom., Washington, DC, 2002, pp. 3731-3736.

[17] D. J. Montana, "The kinematics of multi-fingered manipulation," IEEE Trans. Robot. Autom., vol. 11, no. 4, pp. 491-503, Aug. 1995.

[18] J. K. Salisbury, "Kinematic and force analysis of articulated hands," $\mathrm{Ph} . \mathrm{D}$ dissertation, Dept. Mech. Eng., Stanford Univ., Stanford, CA, 1982.

[19] L. Han and J. C. Trinkle, "The instantaneous kinematics of manipulation," in Proc. IEEE Int. Conf. Robot. Autom., Leuven, Belgium, 1998, pp. 1944 1949. 
[20] B. Kiss, J. Lévine, and B. Lantos, "On motion planning for robotic manipulation with permanent rolling contacts," Int. J. Robot. Res., vol. 21, no. 5/6, pp. 443-461, 2002.

[21] A. Cole, J. E. Hauser, and S. Sastry, "Kinematics and control of multifingered hands with rolling contact," IEEE Trans. Autom. Control, vol. 34, no. 4, pp. 398-404, Apr. 1989.

[22] N. Sarkar, X. Yun, and V. Kumar, "Control of contact interactions with acatastatic nonholonomic constraints," Int. J. Robot. Res., vol. 16, no. 3, pp. 357-374, 1997.

[23] K. Harada and M. Kaneko, "Rolling based manipulation under neighborhood equilibrium," in Proc. IEEE Int. Conf. Robot. Autom, Seoul, Korea, 2001, pp. 2492-2498.

[24] R. Ozawa, S. Arimoto, S. Nakamura, and J. Bae, "Control of an object with parallel surfaces by a pair of finger robots without object sensing," IEEE Trans. Robot., vol. 21, no. 5, pp. 965-976, Oct. 2005.

[25] C. C. Phipps and M. A. Minor, "Quasi-static rolling control of the rolling disk biped robot," in Proc. IEEE Int. Conf. Robot. Autom., Pasadena, CA, 2008, pp. 1239-1245.

[26] J. S. Dai and D. R. Kerr, "Analysis of force distribution in grasps using augmentation," Proc. Inst. Mech. Eng. C, Mech. Eng. Sci., vol. 210, no. C1, pp. 15-22, 1996.

[27] M. P. do Carmo, Differential Geometry of Curves and Surfaces. Englewood Cliffs, NJ: Prentice-Hall, 1976.

[28] E. Cartan, Riemannian Geometry in an Orthogonal Frame. Singapore: World Scientific, 2001.

[29] H. Cartan, Differential Forms. New York: Dover, 1996, pp. 139-163.

[30] M. R. Rosenberg, Analytical Dynamics of Discrete Systems. New York: Plenum, 1977.

[31] F. Bullo and A. D. Lewis, Geometric Control of Mechanical Systems: Modeling, Analysis, and Design for Simple Mechanical Control Systems. New York: Springer-Verlag, 2005. 